\title{
Micelas de caseína: dos monômeros à estrutura supramolecular
}

\section{Casein micelles: from the monomers to the supramolecular structure}

\author{
Naaman Nogueira Silva ${ }^{1 *}$ (D), Federico Casanova², Michele da Silva Pinto ${ }^{3}$ \\ Antônio Fernandes de Carvalho ${ }^{4}$, Frédéric Gaucheron ${ }^{5}$ \\ ${ }^{1}$ Universidade Federal de São Carlos (UFSCar), Centro de Ciências da Natureza, Campina do Monte Alegre/SP - Brasil \\ ${ }^{2}$ Technical University of Denmark (DTU), Food Production Engineering, Lyngby - Denmark \\ ${ }^{3}$ LABOCEA, Plouzané - France \\ ${ }^{4}$ Universidade Federal de Viçosa (UFV), Departamento de Tecnologia de Alimentos, Viçosa/MG - Brasil \\ ${ }^{5}$ Centre National Interprofessionnel de l'Economie Laitiere (CNIEL), Paris - France
}

*Corresponding Author: Naaman Nogueira Silva, Universidade Federal de São Carlos (UFSCar), Centro de Ciências da Natureza, Rua Serafim Libaneo, 4, Centro, Caixa Postal: 64, CEP: 18245-970, Campina do Monte Alegre/SP - Brasil, e-mail: naaman.nogueira@yahoo.com.br

Cite as: Silva, N. N., Casanova, F., Pinto, M. S., Carvalho, A. F., \& Gaucheron, F. (2019). Casein micelles: from the monomers to the supramolecular structure. Brazilian Journal of Food Technology, 22, e2018185. https://doi.org/10.1590/1981-6723.18518

\begin{abstract}
Resumo
A importância primária das micelas de caseína reside no fato de que os processos empregados na transformação do leite em quaisquer de seus derivados dependem, direta ou indiretamente, de sua estabilidade ou de sua desestabilização controlada. Assim, o objetivo do presente trabalho é apresentar uma revisão atualizada sobre a organização estrutural das micelas de caseína. Em termos físico-químicos, as micelas de caseína podem ser definidas como agregados supramoleculares esféricos e porosos, altamente hidratados, carregados negativamente, com diâmetro médio de $200 \mathrm{~nm}$, e que apresentam aproximadamente $10^{4}$ cadeias polipeptídicas. Além de água, as micelas são constituídas por quatro tipos de caseínas, chamadas de $\alpha_{s_{1}}, \alpha_{s_{2}}$, $\beta$, e $k$-caseínas, que estão unidas por meio de interações hidrofóbicas e eletrostáticas, e pela presença de minerais, sobretudo sais de fosfato de cálcio, os quais são os principais responsáveis pela manutenção da estrutura micelar. A estabilidade das micelas de caseína é atribuída à presença de uma camada externa difusa, formada basicamente por k-caseína. Apesar de as propriedades coloidais das micelas de caseína serem conhecidas, ainda não há consenso sobre como as moléculas de caseína estão estruturadas em seu interior. Portanto, os principais modelos que descrevem a organização interna das micelas de caseína são apresentados na parte final do artigo.
\end{abstract}

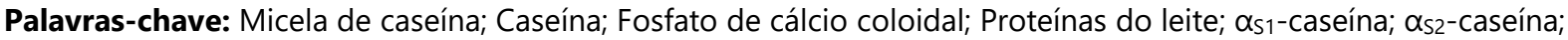
B-caseína; k-caseína.

\section{Abstract}

The most relevant aspect concerning casein micelles lies in the fact that all the procedures employed in the transformation of milk into dairy products depend, directly or indirectly, on their stability or their controlled destabilization. Thus, the present paper aimed to present an updated review of the structural organization of casein 
micelles. Physicochemically, casein micelles can be defined as spherical, porous supramolecular aggregates, highly hydrated, negatively charged, with a mean diameter of about $200 \mathrm{~nm}$, and presenting approximately $10^{4}$ polypeptide chains. Besides water, casein micelles are constituted of four types of casein molecule, namely $\alpha_{\mathrm{S1}}$, $\alpha_{\mathrm{s} 2}, \beta$ and $\mathrm{k}$-caseins, which are held together by means of hydrophobic and electrostatic interactions, and by the presence of minerals, mainly composed of calcium phosphate salts, which are considered the main factor responsible for maintaining the micellar structure. The stability of the casein micelles is attributed to the presence of an outer diffuse layer, basically composed of $\mathrm{k}$-casein. Although the colloidal properties of casein micelles are well known, there is still no consensus concerning their internal structure. Therefore, the main models describing the internal organization of casein micelles are presented in the final part of this article.

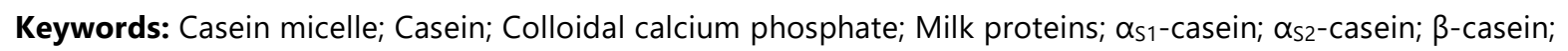
K-casein.

\section{Introdução}

As caseínas são as principais proteínas do leite de vaca (Bos taurus) e representam $80 \%$ do seu conteúdo proteico. Elas podem ser descritas como proteínas fosforiladas, de estrutura aberta e flexível, que atuam como nanoveículos para o transporte de minerais das fêmeas de mamíferos para suas proles, prevenindo a calcificação patológica da glândula mamária (Holt, 2016; Holt et al., 2013).

No leite, as caseínas estão presentes sob a forma de micelas de caseína (MC). As MC são partículas porosas, altamente hidratadas $(\sim 4,0 \mathrm{~mL} / \mathrm{g})$, com diâmetro médio de $200 \mathrm{~nm}$ (Dalgleish, 2011). Elas são constituídas basicamente por moléculas de caseína, água e minerais, sobretudo sais de fosfato de cálcio, os quais atuam como cimento na manutenção da estrutura micelar. As MC se caracterizam como estruturas supramoleculares dinâmicas, que se transformam em função das mudanças nas condições físico-químicas do meio, como pH, temperatura, força iônica, presença de enzimas, etc. (Dalgleish \& Corredig, 2012; Kruif \& Holt, 2003). Ainda não há consenso na comunidade científica sobre a organização interna das MC.

A importância primária das $\mathrm{MC}$ reside no fato de que a aptidão tecnológica do leite para sua transformação em derivados lácteos depende diretamente da estabilidade das MC (Kruif \& Holt, 2003). Em produtos, como leite fluido (pasteurizado ou esterilizado), creme de leite, leite condensado e doce de leite, busca-se a estabilidade das MC. Em outros derivados lácteos, como leites fermentados e queijos, é a desestabilização controlada das MC que permite a fabricação de tais produtos. Adicionalmente, as MC são foco de numerosos estudos que buscam a otimização de suas propriedades tecnológicas e funcionais, como a estabilidade térmica e a aptidão para formação de géis, emulsões e espumas (Broyard \& Gaucheron, 2015). Observa-se também número crescente de publicações que demonstram que as MC podem ser utilizadas como nanoveículos para o transporte de moléculas bioativas, de variadas propriedades químicas, incluindo vitaminas, compostos bioativos e medicamentos para o tratamento de doenças, como o câncer (Ranadheera et al., 2016).

O objetivo do presente trabalho é descrever de forma detalhada a estrutura das MC, com uma abordagem que se inicia pelos seus componentes primários culminando em sua estrutura supramolecular, haja vista o fato de que a literatura em português sobre esse tema é escassa.

\section{Moléculas de caseína}

As caseínas atuam primariamente como fonte de aminoácidos. Entretanto, sabe-se que elas apresentam funções biológicas específicas por atuarem $(i)$ como vetores de cálcio, que passam das fêmeas de mamíferos para os neonatos (Holt et al., 2013); e (ii) como chaperonas, que evitam a formação de agregados proteicos insolúveis, conhecidos como fibrilas amiloides (Holt et al., 2013; Treweek et al., 2011), prevenindo o aparecimento de várias doenças (Rambaran \& Serpell, 2008).

Historicamente, a palavra caseína foi usada para descrever uma classe de proteínas obtidas pela recuperação de precipitado formado após acidificação do leite em pH 4,6 a $20^{\circ} \mathrm{C}$ (O’Mahony \& Fox, 2014). Em termos estruturais, as caseínas são descritas como proteínas reomórficas, com conformação aberta e 
flexível. A ausência de estruturas globulares é explicada pelo alto teor de resíduos prolil (Holt \& Sawyer, 1993). As quatro principais moléculas de caseína são $\alpha_{S 1}, \alpha_{S 2}, \beta$ e $\kappa$-caseínas. As caseínas apresentam diferente composição de aminoácidos (Tabela 1) e uma grande heterogeneidade devido a duas modificações pós-tradução, chamadas de fosforilação e glicosilação (Holland \& Boland, 2014).

Todas as caseínas são fosforiladas. A fosforilação ocorre principalmente nos resíduos seril e, em menor frequência, em resíduos treonil (Holland \& Boland, 2014; Huppertz, 2013). Como consequência da adição de grupamentos fosfato, as caseínas apresentam elevada afinidade por cátions bivalentes, como cálcio e magnésio.

A glicosilação ocorre apenas com as $\kappa$-caseínas e envolve principalmente os resíduos treonil e, em menor extensão, os resíduos seril, localizados na extremidade C-terminal (Kanamori et al., 1980). Os carboidratos envolvidos na glicosilação são galactose, $\mathrm{N}$-acetil-galactosamina e ácido neuramínico, que estão presentes como di, tri ou tetrassacarídeos (Saito \& Itoh, 1992; Tran \& Baker, 1970).

Tabela 1. Composição de aminoácidos de variantes genéticas de $\alpha_{\mathrm{S}^{-}}, \alpha_{\mathrm{S} 2^{-}}, \beta-$ e $\kappa$-caseínas (Huppertz, 2013).

\begin{tabular}{|c|c|c|c|c|}
\hline Aminoácido & $\alpha_{s 1}-C N$ B-8P & $\alpha_{\mathrm{s} 2}-\mathrm{CN}$ A-11P & к-CN A-1P & $\beta-C N A^{2}-5 P$ \\
\hline Asp & 7 & 4 & 3 & 4 \\
\hline Asn & 8 & 14 & 8 & 5 \\
\hline Thr & 5 & 15 & 14 & 9 \\
\hline Ser & 8 & 6 & 12 & 11 \\
\hline SerP & 8 & 11 & 1 & 5 \\
\hline Glu & 25 & 24 & 12 & 19 \\
\hline Gln & 14 & 16 & 14 & 20 \\
\hline Pro & 17 & 10 & 20 & 35 \\
\hline Gly & 9 & 2 & 2 & 5 \\
\hline Ala & 9 & 8 & 15 & 5 \\
\hline $1 / 2$ Cys & 0 & 2 & 2 & 0 \\
\hline Val & 11 & 14 & 11 & 19 \\
\hline Met & 5 & 4 & 2 & 6 \\
\hline Ile & 11 & 11 & 13 & 10 \\
\hline Leu & 17 & 13 & 8 & 22 \\
\hline Tyr & 10 & 12 & 9 & 4 \\
\hline Phe & 8 & 6 & 4 & 9 \\
\hline $\operatorname{Trp}$ & 2 & 2 & 1 & 1 \\
\hline Lys & 14 & 24 & 9 & 11 \\
\hline His & 5 & 3 & 3 & 5 \\
\hline $\operatorname{Arg}$ & 6 & 6 & 5 & 4 \\
\hline Pyr or Glu & 0 & 0 & 1 & 0 \\
\hline Total de resíduos & 199 & 207 & 169 & 209 \\
\hline
\end{tabular}

As letras "A" ou "B" representam variantes genéticas, enquanto que o numeral que precede a letra "P" representa o número de grupamentos fosfato por molécula.

\section{$2.1 \alpha_{s 1}$-caseínas}

As $\alpha_{\mathrm{S} 1}$-caseínas representam 40\% do total de caseínas (Huppertz, 2013). Existem duas principais variantes genéticas, $\mathrm{B}$ e $\mathrm{C}$, que se assemelham quanto às cadeias de aminoácidos e se diferem em seu grau de fosforilação (Mercier et al., 1971).

A referência para a família $\alpha_{\mathrm{S} 1}$ é a variante $\alpha_{\mathrm{S} 1}-\mathrm{CN}$ B-8P (Farrell Junior et al., 2004), sendo a letra B indicativo da sua variante genética e 8P indicativo da presença de oito fosfatos. Ela possui 199 resíduos de aminoácidos, não apresenta resíduo cisteinil e tem massa molar (MM) de 23.615 Da (Farrell Junior et al., 2004). Possui carga de $-21 \mathrm{mV}$ em pH 6,6 e ponto isoelétrico (pI) de 4,91 (baseado na estrutura primária) e de 4,42, incluindo as fosforilações (Farrell Junior et al., 2004; Huppertz, 2013). As ası-caseínas são sensíveis ao cálcio e precipitam na presença de 3-8 $\mathrm{mM} \mathrm{CaCl}_{2} \mathrm{em} \mathrm{pH} \mathrm{7,0} \mathrm{(Aoki} \mathrm{et} \mathrm{al.,} \mathrm{1985).}$ 


\section{$2.2 \alpha_{\mathrm{S} 2}$-caseínas}

As $\alpha_{\mathrm{s} 2}$-caseínas correspondem a $10 \%$ do total de caseínas e possuem níveis variados de fosforilação (10-13 resíduos fosfosseril por molécula) (Huppertz, 2013). A referência é a $\alpha_{\mathrm{s} 2}-\mathrm{CN}$ A-11P (Farrell Junior et al., 2004), que possui uma ponte dissulfeto interna (cisteína) (Rasmussen et al., 1994). Esta caseína possui 207 aminoácidos, MM de 25.226 Da (Farrell Junior et al., 2004), sendo a mais hidrofílica das caseínas (Swaisgood, 2003). Seu pI, baseado na sua estrutura primária, é de 4,91, e incluindo as fosforilações, é de 4,42 (Huppertz, 2013). Como as $\alpha_{\mathrm{s} 2}$-caseínas são as mais fosforiladas, elas são as mais sensíveis ao cálcio. Apenas $2 \mathrm{mM}$ de $\mathrm{CaCl}_{2}$ provocam a precipitação de $90 \%$ das $\alpha_{\mathrm{s} 2}$-caseínas em pH 7,0 (Aoki et al., 1985).

\section{3 $\beta$-caseínas}

A família das $\beta$-caseínas representa $35 \%$ das caseínas, com 0-5 resíduos fosfosseril por molécula (Swaisgood, 2003). A referência é a $\beta-\mathrm{CN} \mathrm{A}^{2}-5 \mathrm{P}$, mas vinte variantes genéticas já foram identificadas (Farrell Junior et al., 2004). Ela contém 209 resíduos, sua MM é 23.983 Da (Dumas et al., 1972). Seu pI, baseado na estrutura primária, é 5,13, e incluindo as fosforilações, é 4,65 (Huppertz, 2013). A $\beta$-caseína é a mais hidrofóbica das caseínas, sendo fortemente anfifílica (Farrell Junior, 2011; Swaisgood, 2003), o que explica sua tendência a formar agregados micelares em solução (Leclerc \& Calmettes, 1997).

As $\beta$-caseínas são menos sensíveis à precipitação na presença de íons Ca que as $\alpha_{\mathrm{s} 1^{-}}$e $\alpha_{\mathrm{s} 2}$-caseínas. A precipitação depende da temperatura: $20 \mathrm{mM} \mathrm{CaCl}_{2}$ induzem a precipitação a $20^{\circ} \mathrm{C}$, enquanto $4 \mathrm{mM} \mathrm{CaCl}_{2}$ têm o mesmo efeito a $40{ }^{\circ} \mathrm{C}$ (Dalgleish \& Parker, 1980).

\section{4 k-caseínas}

As $\kappa$-caseínas representam $15 \%$ das proteínas do leite e se diferenciam das outras caseínas por serem glicosiladas (Swaisgood, 2003). A família da $\kappa-\mathrm{CN}$ apresenta 11 variantes genéticas com diferentes graus de fosforilação (0-3) e glicosilação (Farrell Junior et al., 2004; Huppertz, 2013). A proteína de referência desta família é a א-CN A-1P (Farrell Junior et al., 2004). Ela possui 169 aminoácidos, dois resíduos cisteinil, MM de 19.052 Da e pI de 5,6 (incluindo as fosforilações) (Jollès et al., 1972a, 1972b; Mercier et al., 1972, 1973). No leite, as $\kappa$-caseínas estão presentes como polímeros, de dímeros a octâmeros, que são atribuídos à formação de ligações dissulfeto entre os resíduos cisteinil (Rasmussen et al., 1994). Cerca de 36\% das moléculas de $\kappa$-caseínas não são glicosiladas e os $64 \%$ restantes possuem de 1 a 10 carboidratos por molécula

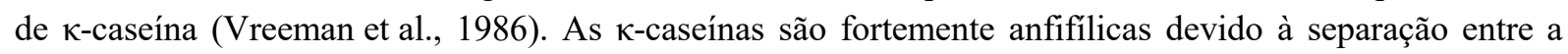
extremidade N-terminal (1-105) hidrofóbica e a extremidade hidrofílica C-terminal (106-169) (Hill \& Wake, 1969), em que os resíduos glicosilados e/ou fosforilados estão presentes. Sua solubilidade não depende da presença de cálcio, o que é resultado do baixo nível de fosforilação (Swaisgood, 2003).

\section{Minerais}

A composição de minerais do leite é complexa e depende de fatores genéticos, estágio de lactação, alimentação e saúde dos animais (Gaucheron, 2004). Os principais minerais do leite estão apresentados Tabela 2. Apesar de ser uma molécula orgânica, o citrato está incluído na composição mineral do leite por participar de seu equilíbrio iônico.

Os minerais do leite podem ser agrupados em duas frações: (i) fração solúvel, representada pelos sais e íons presentes na fase contínua do leite; e (ii) fração coloidal, que inclui os minerais que estão associados às MC (Tabela 2). 
Tabela 2. Composição aproximada de sais no leite, incluindo a distribuição entre frações coloidal e solúvel (Gaucheron, 2005; Lucey \& Horne, 2009).

\begin{tabular}{cccc}
\hline Elementos & Concentração $(\mathbf{m M})$ & Coloidal (\%) & Solúvel (\%) \\
\hline Cátions & & & 31 \\
\hline Cálcio & $26-32$ & 69 & 53 \\
\hline Magnésio & $4-6$ & 47 & 94 \\
Potássio & $31-43$ & 6 & 95 \\
Sódio & $17-28$ & 5 & 95 \\
\hline Ânions & & & 86 \\
\hline Cloreto & $22-34$ & 5 & 35 \\
\hline Citrato & $7-11$ & 14 & 65 \\
\hline Fósforo & $29-32$ & & \\
\hline
\end{tabular}

\subsection{Fração solúvel}

Conhecendo-se a composição da fase contínua do leite e as constantes de associação intrínseca entre os íons presentes, é possível calcular a concentração de sais e íons livres que compõem a fração solúvel do leite, conforme apresentado na Tabela 3.

Tabela 3. Concentrações calculadas de íons e sais presentes na fração solúvel de leite.

\begin{tabular}{cccccc}
\hline \multirow{2}{*}{ Ânions $(\mathbf{m M})$} & \multirow{2}{*}{ Íons livres } & \multicolumn{4}{c}{ Cátions $\mathbf{( m M )}$} \\
\cline { 3 - 6 } & & $\mathbf{C a}^{\mathbf{2 +}}$ & $\mathbf{M g}^{\mathbf{2 +}}$ & $\mathbf{N a}^{+}$ & $\mathbf{K}^{+}$ \\
\hline $\mathrm{H}_{2} \mathrm{Cit}^{-}$ & + & + & + & + & + \\
$\mathrm{Hcit}^{2-}$ & 0,04 & 0,01 & + & + & + \\
$\mathrm{Cit}^{3-}$ & 0,26 & 6,96 & 2,02 & 0,03 & 0,04 \\
$\mathrm{H}_{2} \mathrm{PO}_{4}^{-}$ & 7,50 & 0,07 & 0,04 & 0,10 & 0,18 \\
$\mathrm{HPO}_{4}{ }^{2-}$ & 2,65 & 0,59 & 0,34 & 0,39 & + \\
$\mathrm{PO}_{4}^{3-}$ & + & 0,01 & + & + & 0,68 \\
$\mathrm{Cl}^{-}$ & 30,9 & 0,26 & 0,07 & 0,39 & 36,29 \\
\hline Íons livres & & 2,00 & 0,81 & 20,92 & + \\
\hline
\end{tabular}

Resumido e adaptado de Holt et al. (1981). (+) concentração menor que 0,005 mM.

\subsection{Fração coloidal}

A fração coloidal compreende dois principais tipos de sais: (i) cátions e ânions ligados diretamente aos resíduos de aminoácidos das caseínas; e (ii) fosfato de cálcio coloidal. Quanto ao tipo (i), pode-se afirmar que as caseínas têm alta afinidade por cátions bivalentes, principalmente cálcio, o que é atribuído à presença dos resíduos glutamil, aspartil e, principalmente, aos resíduos fosfosseril. Como regra geral, quanto mais fosforilada for uma molécula de caseína, maior será sua capacidade de se ligar ao cálcio (Dalgleish \& Parker, 1980; Holt et al., 1981; Parker \& Dalgleish, 1981; White \& Davies, 1958). Antes de abordar o tipo (ii), é necessário considerar a baixa solubilidade do fosfato de cálcio $\left(\mathrm{CaHPO}_{4}\right)$, que é de $0,59 \mathrm{mM}$ no $\mathrm{pH}$ do leite (pH 6,7) (Gaucheron, 2004). Portanto, durante a síntese do leite na glândula mamária, a fase contínua fica supersaturada em $\mathrm{CaHPO}_{4}$ (Holt, 1981). Consequentemente, o estado termodinâmico mais estável é alcançado pela nucleação e precipitação dos sais supersaturados (Walstra, 2002). Kruif \& Holt (2003) postularam que a nucleação ocorre sobre as sequências de resíduos fosfosseril das caseínas devido à sua afinidade química pelos sais precipitados. Estes precipitados são chamados de fosfato de cálcio coloidal (FCC) (Kruif \& Holt, 2003; Holt et al., 1998). Para que ocorra a formação de FCC, é necessária a presença de uma sequência de pelo menos três resíduos fosfosseril por molécula de caseína (Aoki et al., 1992).

Os minerais presentes na fase coloidal do leite estão em equilíbrio dinâmico com os minerais em sua fase contínua (Figura 1). Este equilíbrio é dependente de condições físico-químicas do meio, como temperatura, pH e força iônica. 

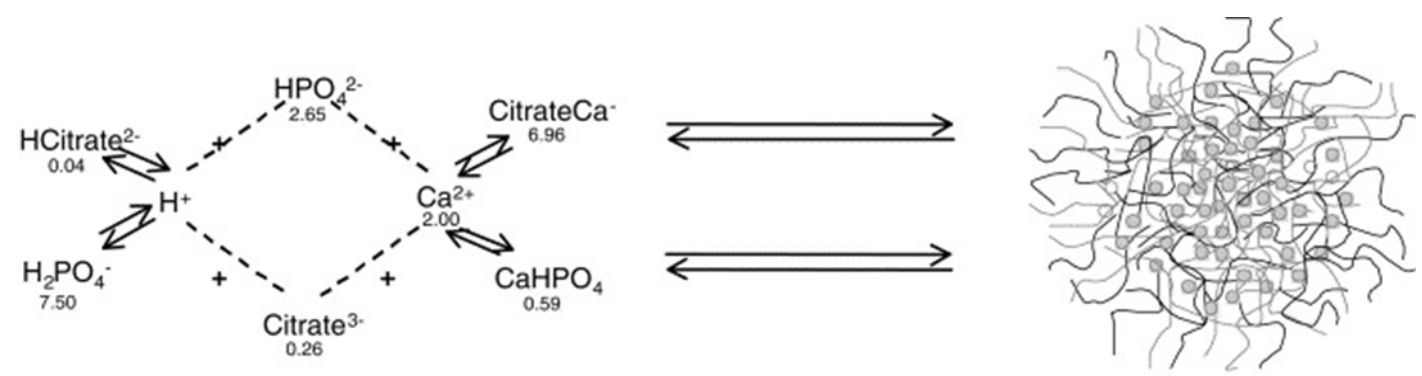

Figura 1. Equilíbrio dinâmico dos minerais de leite entre as fases contínua e coloidal. As concentrações são mostradas em mM, logo abaixo das espécies iônicas. Imagem reproduzida de Philippe et al. (2005), com permissão de Elsevier.

\subsubsection{Fosfato de Cálcio Coloidal (FCC)}

O FCC é constituído principalmente por fósforo e cálcio, mas também apresenta concentrações significativas de citrato e magnésio. A razão molar aproximada de $\mathrm{Ca} / \mathrm{P}$ é de $\sim 1,61$, citrato/P de $\sim 0,097 \mathrm{e}$ $\mathrm{Mg} / \mathrm{P}$ de 0,044 (Holt, 1982). A natureza física do FCC continua controversa e já foi descrita como sendo básica, tipo apatita $\left(\mathrm{Ca}_{3}\left(\mathrm{PO}_{4}\right)\right.$ (Pyne \& McGann, 1960), ou mais ácida, como bruchita $\left(\mathrm{CaHPO}_{4}\right)$ (Holt et al., 1982), e ainda como um precipitado amorfo (McGann et al., 1983). Recentemente, foi descoberta uma nova estrutura de fosfato de cálcio no interior das MC (Hindmarsh \& Watkinson, 2017). Essa nova forma ainda não foi descrita na literatura, mas os resultados indicam ligações entre resíduos fosfosseril intermediadas por cálcio.

Segundo Kruif \& Holt (2003), a fórmula química do FCC é (Equação 1):

$\left[\mathrm{Ca}_{13,2}(\mathrm{Pi})_{6,5} \mathrm{Mg}_{1,0} \mathrm{Cit}_{1,3} \mathrm{SerP}_{4} \mathrm{Cas}\right]_{49}$

em que $\mathrm{SerP}_{4}$ é um peptídeo fosforilado e Cit é citrato.

Holt et al. (1998) analisaram o FCC por espalhamento de raios X e nêutrons, e propuseram um modelo no qual o FCC é constituído por um núcleo esférico de 2,31 nm, envolto por 50 moléculas de caseínas, formando uma capa com raio de 4,04 nm. Análise por criomicroscopia eletrônica (Marchin et al., 2007) revelou o FCC como regiões eletronicamente densas de aproximadamente $3 \mathrm{~nm}$, distribuídas no interior das MC (Figura 2). Segundo Kruif \& Holt (2003) e Kruif et al. (2012), uma MC típica contém algumas centenas de núcleos de FCC.

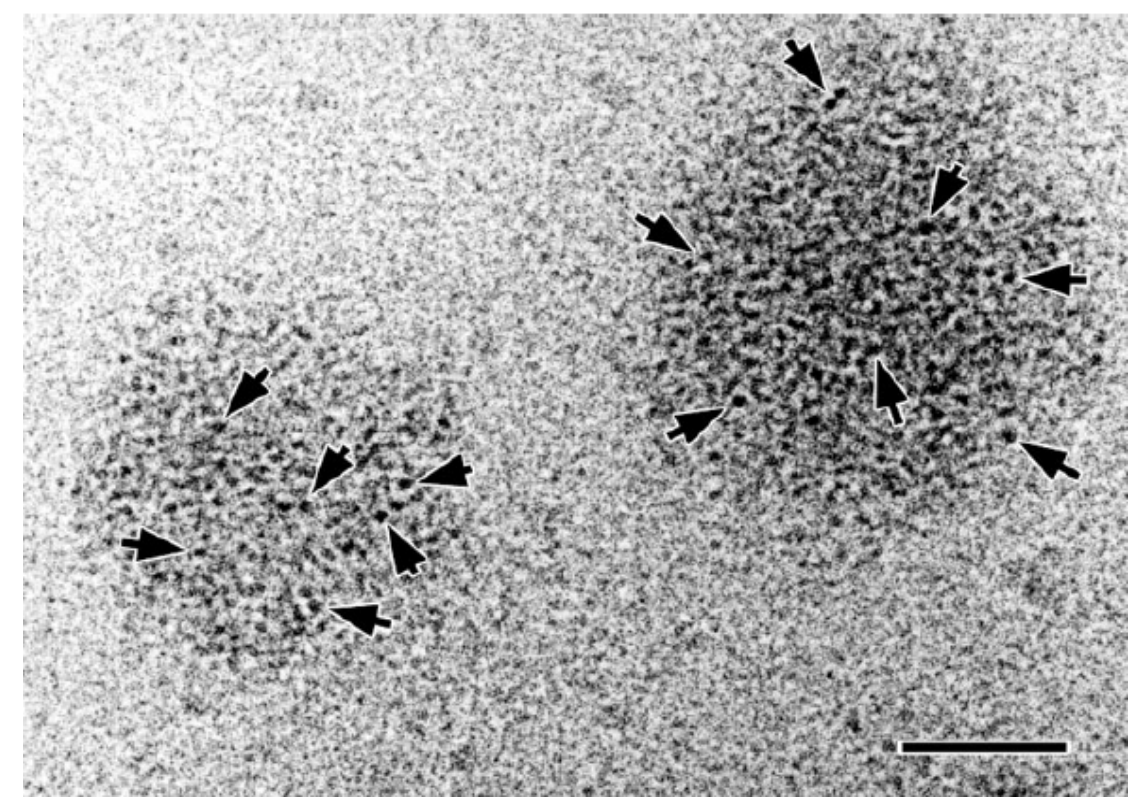

Figura 2. Criomicroscopia eletrônica de micelas de caseína. A escala da barra é de $50 \mathrm{~nm}$. As setas apontam para núcleos de fosfato de cálcio coloidal. Imagem reproduzida de Marchin et al. (2007), com permissão de AIP Publishing. 


\section{Micelas de Caseína (MC)}

\subsection{Características gerais das MC}

No leite, as MC estão naturalmente presentes como partículas polidispersas, de aproximadamente $200 \mathrm{~nm}$ de diâmetro, constituídas de moléculas de caseínas, sais (principalmente fosfato de cálcio) e água. Elas são altamente hidratadas $\left(4,0 \mathrm{~g} \mathrm{H}_{2} \mathrm{O} \mathrm{g}^{-1}\right.$ proteína), sua composição mineral representa $6 \%$ a $8 \%$ da matéria seca e elas contêm $\sim 10^{4}$ moléculas de caseínas por MC (Dalgleish, 2011; Kruif \& Holt, 2003). De acordo com (Jones et al., 2012) (Recomendações - IUPAC 2013), as MC podem ser descritas como agregados supramoleculares que são mantidos juntos pela ação de interações intermoleculares (não covalentes). As principais características das MC estão descritas na Tabela 4.

Tabela 4. Características gerais das micelas de caseínas extraídas e adaptadas de (McMahon \& Brown, 1984).

\begin{tabular}{|c|c|}
\hline Características & Valores \\
\hline Diâmetro & $200 \mathrm{~nm}(50-500 \mathrm{~nm})$ \\
\hline Área superficial & $8 \times 10^{-10} \mathrm{~cm}^{2}$ \\
\hline Volume & $2,1 \times 10^{-15} \mathrm{~cm}^{3}$ \\
\hline Densidade (hidratada) & $1,0632 \mathrm{~g} \mathrm{~cm}^{3}$ \\
\hline Massa & $2,2 \times 10^{-15} \mathrm{~g}$ \\
\hline Conteúdo de água & $63 \%$ \\
\hline Hidratação & $3,7 \mathrm{~g} \mathrm{H}_{2} \mathrm{O} \mathrm{g}^{-1}$ \\
\hline Voluminosidade & $4,4 \mathrm{~cm}^{3} \mathrm{~g}^{-1}$ \\
\hline Massa molar (hidratada) & $1,3 \times 10^{9} \mathrm{Da}$ \\
\hline Massa molar (desidratada) & $5 \times 10^{8} \mathrm{Da}$ \\
\hline Número de cadeias peptídicas & $5 \times 10^{3}$ \\
\hline Número de partículas por $\mathrm{mL}$ de leite & $10^{14}-10^{16}$ \\
\hline Superfície das micelas por $\mathrm{mL}$ de leite & $5 \times 10^{4} \mathrm{~cm}^{2}$ \\
\hline Distância média livre & $240 \mathrm{~nm}$ \\
\hline
\end{tabular}

\subsection{Tamanho das MC}

O uso de técnicas não invasivas, como o espalhamento de luz, permitiu uma estimativa correta do tamanho e da polidispersidade das MC. Kruif (1998) observou que as MC apresentam uma distribuição log-normal, com diâmetro variando de $\sim 80$ a $550 \mathrm{~nm}$ e diâmetro médio de $200 \mathrm{~nm}$. Assumindo uma distribuição Gaussiana, as MC exibiram uma polidispersidade de 50\%. Embora as MC sejam frequentemente descritas como partículas polidispersas, quando o leite é oriundo de uma única vaca, as MC são quase monodispersas; observe-se que seu diâmetro não é influenciado pelo período de lactação e por lactações consecutivas (Kruif \& Huppertz, 2012).

\subsection{Voluminosidade das MC}

Voluminosidade é o volume hidrodinâmico ocupado por unidade de massa da partícula. Em sistemas não diluídos, em que as partículas estão interagindo, como no caso do leite, a voluminosidade das MC pode ser obtida pela equação de Krieger-Dougherty (Krieger \& Dougherty, 1959) (Equação 2):

$\eta=\eta_{s}\left(1-\varphi / \varphi_{\max }\right)^{[2,5] \varphi_{\max }}$

em que $\eta$ é a viscosidade da suspensão, $\eta_{\mathrm{s}}$ é a viscosidade do solvente e $\varphi_{\max }$ é a fração de volume máxima que se pode atingir, que é $\sim 0,78$ para suspensão de MC (Bouchoux et al., 2009). Utilizando esta equação, a voluminosidade das MC está em torno de 4-5 mL.g-1 (Nöbel et al., 2012, 2016). 


\subsection{Superfície das MC e localização da k-caseína}

Microscopias de força atômica (Ouanezar et al., 2012) e eletrônica de varredura (Dalgleish et al., 2004) permitiram obter uma visão detalhada da superfície das MC. Por meio dessas técnicas, as MC são vistas como partículas esféricas, variando de $\sim 80$ a $400 \mathrm{~nm}$ de diâmetro, que exibem superfície irregular e rugosa (Figura 3).
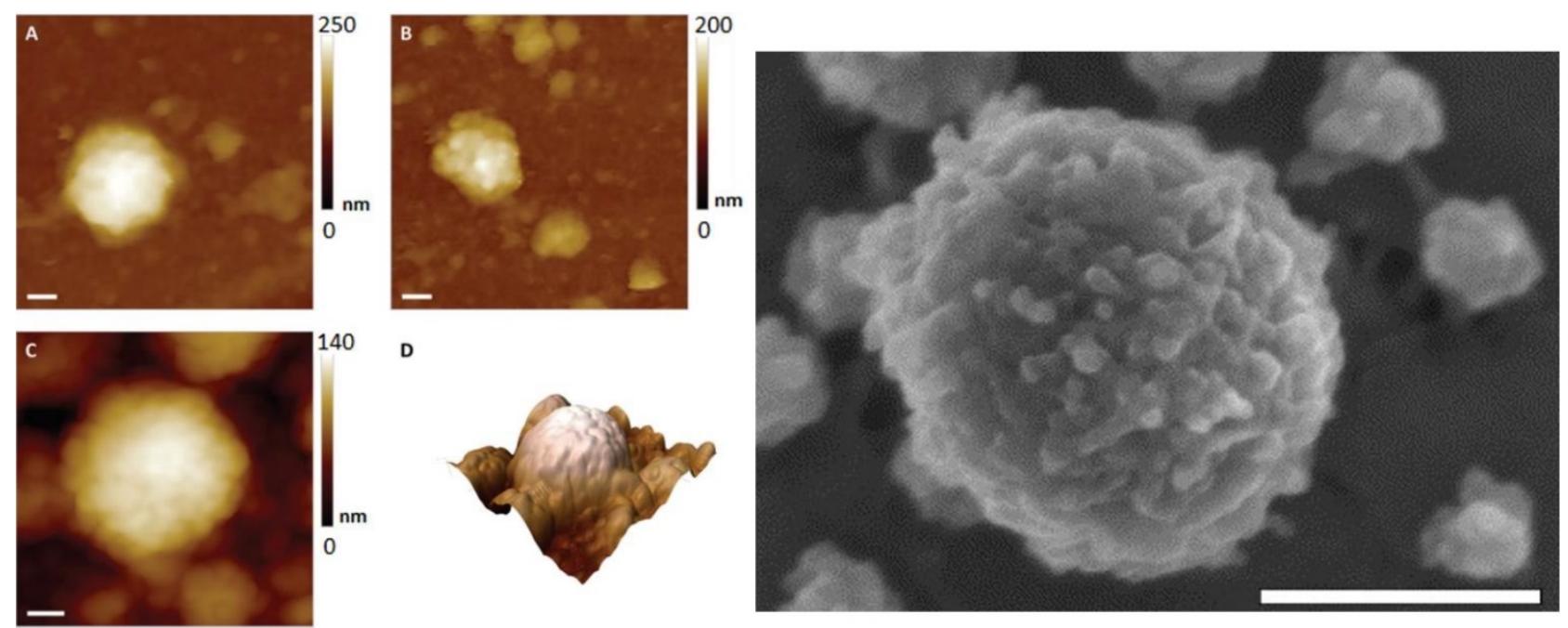

D

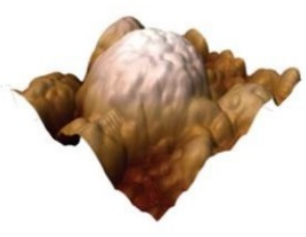

Figura 3. (Esquerda) (A), (B), (C) são imagens de microscopia de força atômica de micelas de caseína em pH 6,7; (D) imagem tridimensional da imagem C. A barra de escala é de $100 \mathrm{~nm}$. Imagens reproduzidas de Ouanezar et al. (2012), com permissão de American Chemical Society. (Direita) Imagem de microscopia eletrônica de varredura de micelas de caseína em pH 7,0. A barra de escala corresponde a $200 \mathrm{~nm}$. Imagem reproduzida de Dalgleish et al. (2004), com permissão de Elsevier.

A composição em moléculas de caseína não é homogênea do interior para o exterior das MC. Waugh \& Von Hippel (1956) foram os primeiros a sugerir que as MC são estabilizadas por uma camada externa de К-caseína, uma vez que essas caseínas são solúveis nas concentrações de cálcio encontradas no leite, e são prontamente hidrolisadas pela quimosina (enzima utilizada para coagulação do leite durante a produção de queijo). Outros estudos (Dalgleish et al., 1989; Donnelly et al., 1984) mostraram que quando as MC são fracionadas de acordo com seu tamanho, sua composição relativa em caseínas $\alpha_{\mathrm{s} 1^{-}}, \alpha_{\mathrm{s} 2^{-}}$e $\beta$ permanece constante, enquanto que o conteúdo de $\kappa$-caseína é inversamente proporcional ao tamanho das MC. Estes resultados confirmaram que a $\kappa$-caseína está localizada na superfície, uma vez que a relação superfície/volume de uma esfera é inversamente proporcional ao seu raio.

\subsection{Estabilidade das MC}

Holt \& Dalgleish (1986) propuseram que as MC são estabilizadas por uma camada externa difusa, composta pela extremidade N-terminal das $\kappa$-caseínas, cujo comprimento foi calculado como sendo de $12 \mathrm{~nm}$ (Figura 4B). Essa camada é responsável pela carga negativa das MC, apresentando valores de aproximadamente -20 mV na fase contínua do leite (Dalgleish, 2011; Darling \& Dickson, 1979). A carga negativa é resultado da presença de resíduos aspartil e glutamil, e moléculas de ácido neuramínico ligadas à К-caseína, que estão ionizados no pH do leite (Kruif \& Holt, 2003; Swaisgood, 2003).

Embora as cargas negativas das MC contribuam para sua estabilidade, elas não são suficientes para prevenir a agregação entre micelas (Walstra, 2002). Segundo a teoria DLVO (de Derjaguin, Landau, Verwey e Overbeek), o potencial de interação entre duas partículas, separadas por uma distância $d$, pode ser estimado pela soma das repulsões eletrostáticas e forças atrativas de van der Waals (Mezzenga \& Fischer, 2013). Tuinier \& Kruif (2002) desenvolveram um modelo para predizer a estabilidade das MC considerando também 
a contribuição estérica da camada externa das $\kappa$-caseínas. Assim, o potencial de interação entre duas $\mathrm{MC}$ deve considerar o impedimento estérico ("brush repulsion”), a repulsão eletrostática e a atração de van der Waals (Figura 4A). Segundo os autores, a estabilidade das MC é atribuída principalmente à contribuição estérica da camada externa de $\kappa$-caseína, que foi descrita como extremidades eletricamente carregadas (“salted brush") (Figura 4B).

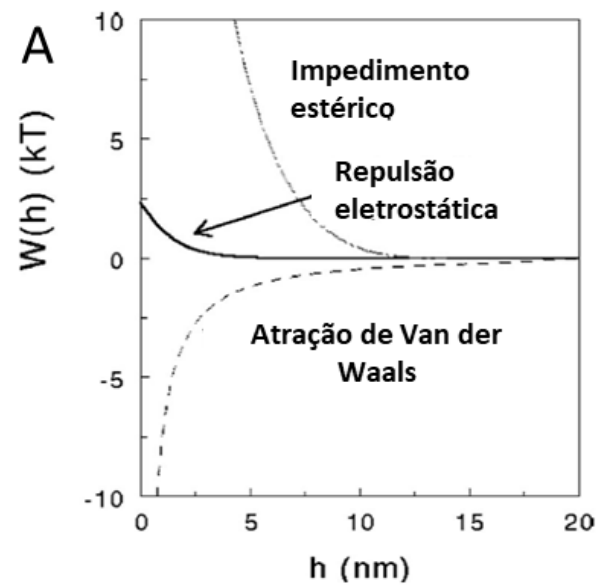

\section{B Extremidades eletricamente carregadas}

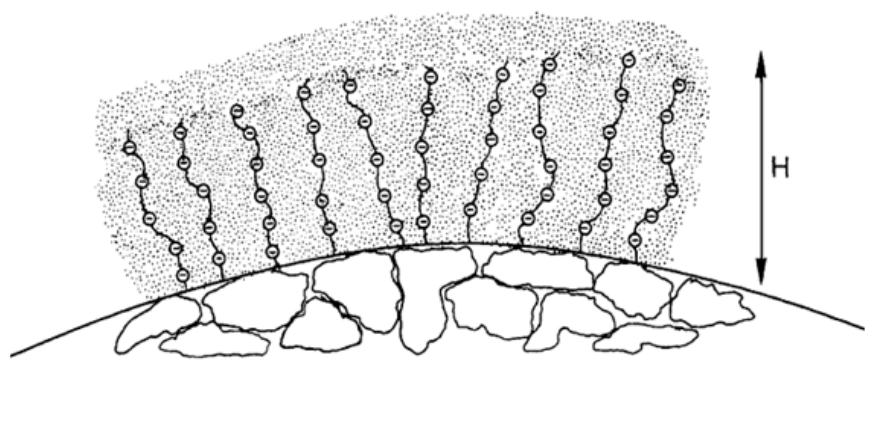

Figura 4. (A) Contribuição do potencial de interação [W(h) kT] entre duas micelas de caseína devido ao impedimento estérico, repulsão eletrostática e atração de van der Waals em função da distância (h) em pH 6,7. Imagem reproduzida de Tuinier \& Kruif (2002), com permissão de AIP Publishing; (B) Representação das extremidades eletricamente carregadas de $\kappa$-caseínas ("salted brush"), com comprimento representado pela letra (H). Imagem reproduzida Kruif (1999), com permissão de Elsevier.

\section{Organização interna e modelos de MC}

Desde os anos 1960, há um debate científico sobre a organização interna das MC. Neste trabalho, será apresentada uma breve retrospectiva dos principais modelos propostos para a estrutura interna das MC.

\subsection{Modelo de submicelas}

Inicialmente, o modelo de submicelas foi baseado na observação das MC por meio de microscopia eletrônica. Nesta técnica, as MC apresentam um formato esférico contendo subunidades de aproximadamente $20 \mathrm{~nm}$, que foram interpretadas como submicelas (Buchheim \& Welsch, 1973; Rose \& Colvin, 1966; Shimmin \& Hill, 1964). Segundo este modelo, a к-caseína localiza-se na superfície das submicelas e o FCC atua como agente de ligação entre as submicelas (Morr, 1967; Slattery, 1976; Slattery \& Evard, 1973). Estudos utilizando espalhamento de nêutrons a baixos ângulos (SANS) mostraram uma inflexão na curva de espalhamento, correspondendo a uma escala de comprimento de $\sim 18 \mathrm{~nm}$, que foi atribuída à existência das submicelas (Stothart, 1989; Stothart \& Cebula, 1982). Walstra (1999) redesenhou o modelo de submicelas, adicionando os núcleos de FCC no centro das submicelas (Figura 5). 

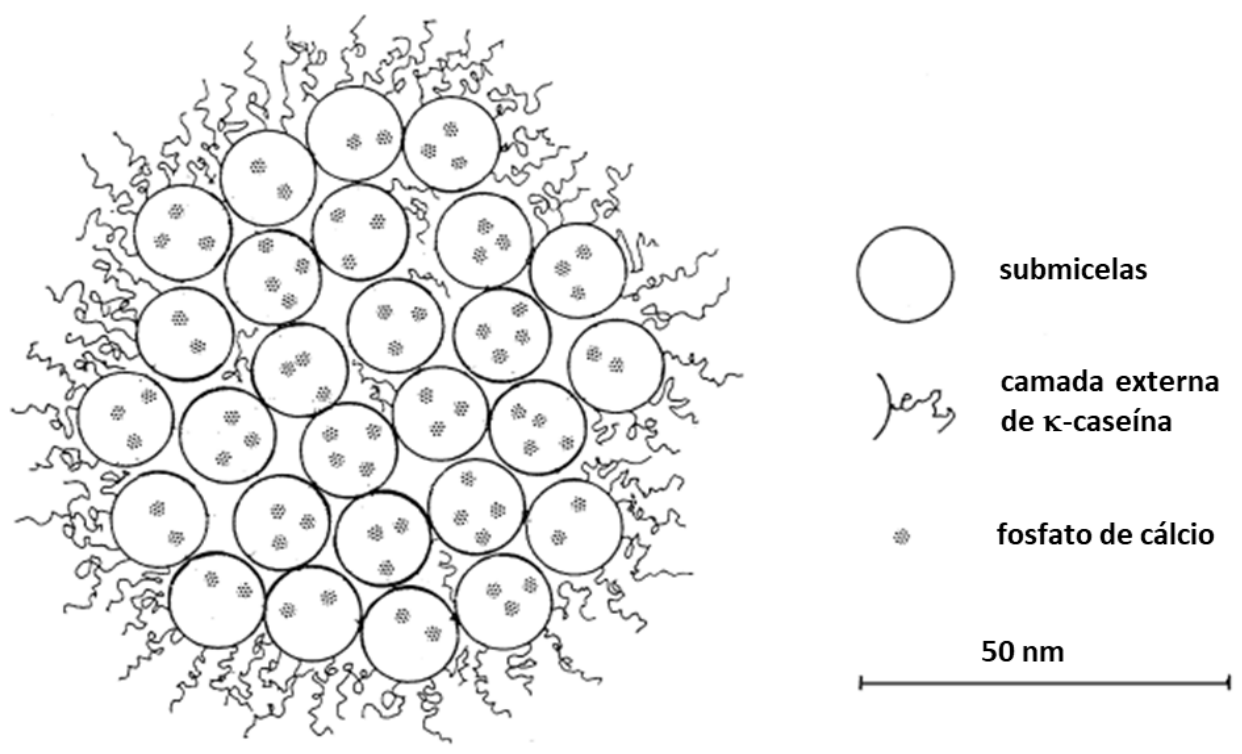

Figura 5. Modelo de submicelas proposto por Walstra (1999). Imagem reproduzida com permissão de Elsevier.

\subsection{Modelo de esponja}

Bouchoux et al. (2010) propuseram um modelo de MC semelhante a uma estrutura de esponja (Figura 6). Estes autores usaram espalhamento de raios $\mathrm{X}$ a baixos ângulos (SAXS) em amostras de concentrações crescentes de $\mathrm{MC}$ e observaram que as MC têm uma estrutura compressível, com três níveis hierárquicos em sua organização. $\mathrm{O}$ nível mais alto seria a própria $\mathrm{MC}$, com dimensão superior a $100 \mathrm{~nm}$, que pode ser comprimida sob estresse osmótico. O nível intermediário consistiria de regiões incompressíveis (densas), de 10 a $40 \mathrm{~nm}$, distribuídas no interior das MC. O nível mais elementar corresponderia aos núcleos de FCC, de 4 a $5 \mathrm{~nm}$, localizados dentro das regiões intermediárias. Posteriormente, Ingham et al. (2016) utilizaram SAXS e empregaram novos modelos matemáticos para interpretação dos dados. Os autores também observaram os três níveis hierárquicos propostos por Bouchoux et al. (2010). Estes resultados são consistentes com observações de MC por criotomografia eletrônica de transmissão (Trejo et al., 2011), nas quais foram detectados canais (> $5 \mathrm{~nm})$ e cavidades $(20-30 \mathrm{~nm})$ preenchidas por água.

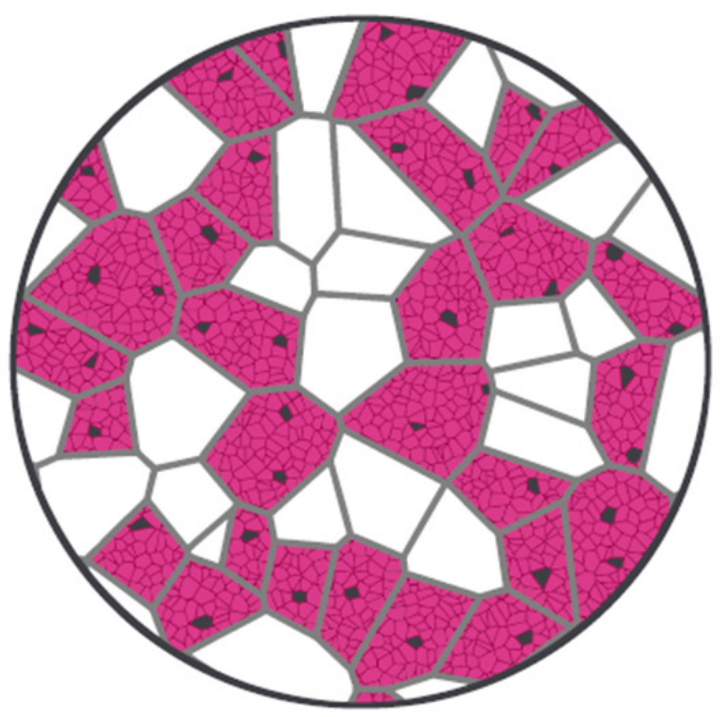

vazio

região

densa

fosfato de

cálcio

$50 \mathrm{~nm}$

Figura 6. Modelo de esponja segundo Bouchoux et al. (2010). Imagem reproduzida com permissão de Elsevier. 


\subsection{Modelo de nanoclusters}

O modelo de "nanoclusters" foi proposto por Kruif \& Holt (2003), Kruif et al. (2012) e Kruif (2014). Segundo esse modelo, as MC são partículas com matriz proteica homogênea, nas quais os núcleos de FCC, i.e., "nanoclusters", estão distribuídos aleatoriamente em seu interior, não havendo organização em submicelas (Figura 7).

Segundo estudos de Holt, de Kruif e colaboradores (Kruif et al., 2012; Kruif \& Holt, 2003; Holt et al., 1998; Tuinier \& Kruif, 2002), a estrutura das MC pode ser descrita da seguinte forma: os núcleos de FCC são circundados por resíduos fosfosseril das $\alpha_{\mathrm{S} 1}, \alpha_{\mathrm{S} 2}$ e $\beta$-caseínas, formando as unidades básicas das MC. Desta forma, as MC são constituídas por várias unidades básicas, que estão conectadas por meio de interações fracas (interações hidrofóbicas, de hidrogênio e iônica, atração eletrostática e de van der Waals) entre as caudas de caseínas de diferentes unidades básicas. O processo de autoassociação entre as unidades básicas é finalizado quando as moléculas de $\kappa$-caseína se depositam sobre a superfície desses conglomerados, formando as MC.

Vale ressaltar que, independentemente do modelo, a estrutura interna das MC não depende de seu tamanho, nem das variantes genéticas das moléculas de caseína (Day et al., 2017), e que os dados da literatura são convergentes quanto à distribuição aleatória dos núcleos de FCC no interior da $\mathrm{MC}$ e à ausência desses componentes na camada externa de $\kappa$-caseína (Bouchoux et al., 2015).

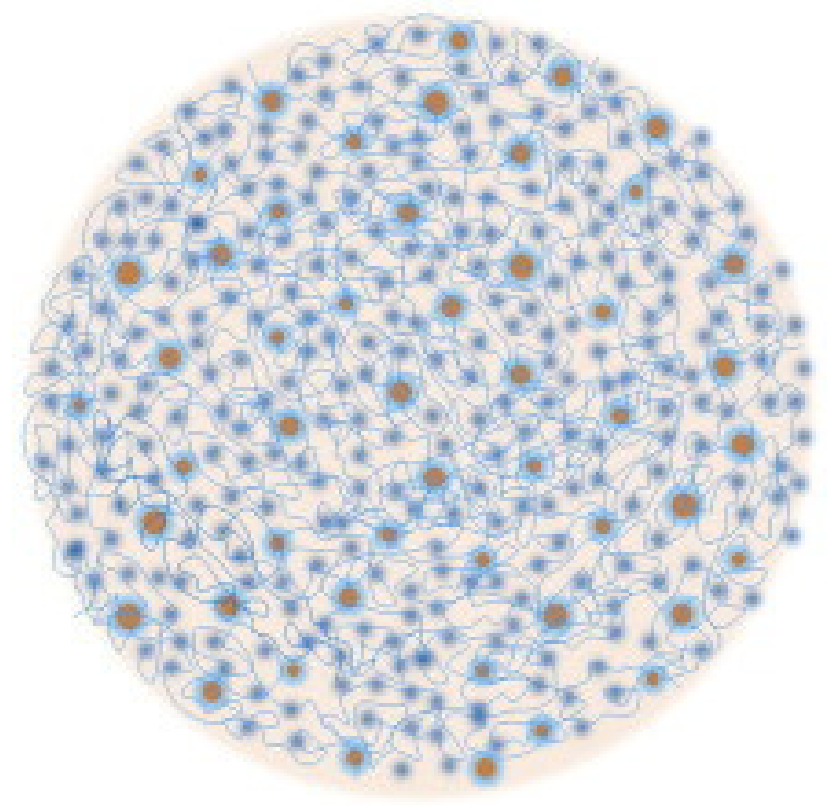

Figura 7. Modelo de nanoclusters de acordo com Kruif et al. (2012). Os pontos escuros representam núcleos de fosfato de cálcio coloidal (FCC). Imagem reproduzida com permissão de Elsevier.

\section{Conclusão}

As MC são estruturas dinâmicas que evoluem em função das condições físico-químicas do meio. Apesar de sua estrutura interna ser controversa, suas propriedades físico-químicas já foram devidamente elucidadas. $\mathrm{O}$ conhecimento da organização das MC é importante na medida em que permite que suas propriedades tecnológicas sejam positivamente modificadas pela indústria de alimentos, além de tornar possível seu uso como partícula nanotransportadora de componente bioativos. 


\section{Referências}

Aoki, T., Toyooka, K., \& Kako, Y. (1985). Role of phosphate groups in the calcium sensitivity of as2-casein. Journal of Dairy Science, 68(7), 1624-1629. http://dx.doi.org/10.3168/jds.S0022-0302(85)81005-5

Aoki, T., Umeda, T., \& Kako, Y. (1992). The least number of phosphate groups for crosslinking of casein by colloidal calcium phosphate. Journal of Dairy Science, 75(4), 971-975. PMid:1578034. http://dx.doi.org/10.3168/jds.S0022-0302(92)77838-2

Bouchoux, A., Debbou, B., Gesan-Guiziou, G., Famelart, M. H., Doublier, J. L., \& Cabane, B. (2009). Rheology and phase behavior of dense casein micelle dispersions. The Journal of Chemical Physics, 131(16), 165106. PMid:19894981. http://dx.doi.org/10.1063/1.3245956

Bouchoux, A., Gésan-Guiziou, G., Perez, J., \& Cabane, B. (2010). How to squeeze a sponge: Casein micelles under osmotic stress, a SAXS study. Biophysical Journal, 99(11), 3754-3762. PMid:21112300. http://dx.doi.org/10.1016/j.bpj.2010.10.019

Bouchoux, A., Ventureira, J., Gesan-Guiziou, G., Garnier-Lambrouin, F., Qu, P., Pasquier, C., Pezennec, S., Schweins, R., \& Cabane, B. (2015). Structural heterogeneity of milk casein micelles: A SANS contrast variation study. Soft Matter, 11(2), 389399. PMid:25388767. http://dx.doi.org/10.1039/C4SM01705F

Broyard, C., \& Gaucheron, F. (2015). Modifications of structures and functions of caseins: A scientific and technological challenge. Dairy Science \& Technology, 95(6), 831-862. http://dx.doi.org/10.1007/s13594-015-0220-y

Buchheim, W., \& Welsch, U. (1973). Evidence for the submicellar composition of casein micelles on the basis of electron microscopical studies. Nederlands Melk-en Zuiveltijdschrift, 27, 163-180.

Dalgleish, D. G. (2011). On the structural models of bovine casein micelles: Review and possible improvements. Soft Matter, 7(6), 2265-2272. http://dx.doi.org/10.1039/C0SM00806K

Dalgleish, D. G., \& Corredig, M. (2012). The structure of the casein micelle of milk and its changes during processing. Annual Review of Food Science and Technology, 3(1), 449-467. PMid:22385169. http://dx.doi.org/10.1146/annurev-food-022811101214

Dalgleish, D. G., \& Parker, T. G. (1980). Binding of calcium ions to bovine asl-casein and precipitability of the protein-calcium ion complexes. The Journal of Dairy Research, 47(1), 113-122. http://dx.doi.org/10.1017/S002202990002094X

Dalgleish, D. G., Horne, D. S., \& Law, A. J. R. (1989). Size-related differences in bovine casein micelles. Biochimica et Biophysica Acta (BBA) - General Subjects, 991(3), 383-387. http://dx.doi.org/10.1016/0304-4165(89)90061-5

Dalgleish, D. G., Spagnuolo, P. A., \& Goff, H. D. (2004). A possible structure of the casein micelle based on high-resolution field-emission scanning electron microscopy. International Dairy Journal, 14(12), 1025-1031. http://dx.doi.org/10.1016/j.idairyj.2004.04.008

Darling, D. F., \& Dickson, J. (1979). The determination of the zeta potential of casein micelles. The Journal of Dairy Research, 46(2), 329-332. http://dx.doi.org/10.1017/S0022029900017258

Day, L., Raynes, J. K., Leis, A., Liu, L. H., \& Williams, R. P. W. (2017). Probing the internal and external micelle structures of differently sized casein micelles from individual cows milk by dynamic light and small-angle X-ray scattering. Food Hydrocolloids, 69, 150-163. http://dx.doi.org/10.1016/j.foodhyd.2017.01.007

Donnelly, W. J., McNeill, G. P., Buchheim, W., \& McGann, T. C. A. (1984). A comprehensive study of the relationship between size and protein composition in natural bovine casein micelles. Biochimica et Biophysica Acta, 789(2), 136-143. PMid:6477926. http://dx.doi.org/10.1016/0167-4838(84)90197-3

Dumas, B. R., Brignon, G., Grosclaude, F., \& Mercier, J. C. (1972). Structure primaire de la caseine beta bovine: Sequence complete. European Journal of Biochemistry, 25(3), 505-514. PMid:4557764. http://dx.doi.org/10.1111/j.14321033.1972.tb01722.x

Farrell Junior, H. M. (2011). Milk proteins: Casein nomenclature, structure, and association. In J. W. Fuquay (Ed.), Encyclopedia of dairy sciences (2nd ed., pp. 765-771). Oxford: Elsevier. http://dx.doi.org/10.1016/B978-0-12-374407-4.00430-1.

Farrell Junior, H. M., Jimenez-Flores, R., Bleck, G. T., Brown, E. M., Butler, J. E., Creamer, L. K., Hicks, C. L., Hollar, C. M., NgKwai-Hang, K. F., \& Swaisgood, H. E. (2004). Nomenclature of the proteins of cows' milk: Sixth revision. Journal of Dairy Science, 87(6), 1641-1674. PMid:15453478. http://dx.doi.org/10.3168/jds.S0022-0302(04)73319-6

Gaucheron, F. (2004). Interactions caséines-cations. In F. Gaucheron (Ed.), Minéraux et produits laitiers (pp. 81-112). Paris: Lavoisier.

Gaucheron, F. (2005). The minerals of milk. Reproduction, Nutrition, Development, 45(4), 473-483. PMid:16045895. http://dx.doi.org/10.1051/rnd:2005030

Hill, R. J., \& Wake, R. G. (1969). Amphiphile nature of K-casein as the basis for its micelle stabilizing property. Nature, 221(5181), 635-639. PMid:5818473. http://dx.doi.org/10.1038/221635a0

Hindmarsh, J. P., \& Watkinson, P. (2017). Experimental evidence for previously unclassified calcium phosphate structures in the casein micelle. Journal of Dairy Science, 100(9), 6938-6948. PMid:28690066. http://dx.doi.org/10.3168/jds.2017-12623

Holland, J. W., \& Boland, M. J. (2014). Post-translational modifications of caseins. In M. Boland, H. Singh \& A. Thompson (Eds.), Milk proteins (2nd ed., pp. 141-168). London: Elsevier.

Holt, C. (1981). Some principles determining salt composition and partitioning of ions in milk. Journal of Dairy Science, 64(10), 1958-1964. http://dx.doi.org/10.3168/jds.S0022-0302(81)82797-X

Holt, C. (1982). Inorganic constituents of milk III. The colloidal calcium phosphate of cow's milk. The Journal of Dairy Research, 49(1), 29-38. PMid:6804550. http://dx.doi.org/10.1017/S002202990002210X 
Holt, C. (2016). Casein and casein micelle structures, functions and diversity in 20 species. International Dairy Journal, 60, 2-13. http://dx.doi.org/10.1016/j.idairyj.2016.01.004

Holt, C., \& Dalgleish, D. G. (1986). Electrophoretic and hydrodynamic properties of bovine casein micelles interpreted in terms of particles with an outer hairy layer. Journal of Colloid and Interface Science, 114(2), 513-524. http://dx.doi.org/10.1016/00219797(86)90437-6

Holt, C., \& Sawyer, L. (1993). Caseins as rheomorphic proteins: Interpretation of primary and secondary structures of the $\alpha_{\mathrm{S} 1}-$ $\beta$ - and k-caseins. Journal of the Chemical Society, Faraday Transactions, 89(15), 2683-2692. http://dx.doi.org/10.1039/FT9938902683

Holt, C., Carver, J. A., Ecroyd, H., \& Thorn, D. C. (2013). Invited review: Caseins and the casein micelle: Their biological functions, structures, and behavior in foods. Journal of Dairy Science, 96(10), 6127-6146. PMid:23958008. http://dx.doi.org/10.3168/jds.2013-6831

Holt, C., Dalgleish, D. G., \& Jenness, R. (1981). Calculation of the ion equilibria in milk diffusate and comparison with experiment. Analytical Biochemistry, 113(1), 154-163. PMid:7270880. http://dx.doi.org/10.1016/0003-2697(81)90059-2

Holt, C., Hasnain, S. S., \& Hukins, D. W. L. (1982). Structure of bovine milk calcium phosphate determined by X-ray absorption spectroscopy. Biochimica et Biophysica Acta (BBA) - General Subjects, 719(2), 299-303. PMid:7150642. http://dx.doi.org/10.1016/0304-4165(82)90102-7

Holt, C., Timmins, P., Errington, N., \& Leaver, J. (1998). A core shell model of calcium phosphate nanoclusters stabilized by b casein phosphopeptides, derived from sedimentation equilibrium and small angle $\mathrm{X}$ ray and neutron scattering measurements. European Journal of Biochemistry, 252(1), 73-78. PMid:9523714. http://dx.doi.org/10.1046/j.1432-1327.1998.2520073.x

Huppertz, T. (2013). Chemistry of the caseins. In P. L. H. McSweeney \& P. F. Fox (Eds.). Advanced dairy chemistry (Vol. 1A, 4th ed., pp. 135-160). Boston: Springer US. http://dx.doi.org/10.1007/978-1-4614-4714-6_4

Ingham, B., Smialowska, A., Erlangga, G. D., Matia-Merino, L., Kirby, N. M., Wang, C., Haverkamp, R. G., \& Carr, A. J. (2016). Revisiting the interpretation of casein micelle SAXS data. Soft Matter, 12(33), 6937-6953. PMid:27491477. http://dx.doi.org/10.1039/C6SM01091A

Jollès, J., Schoentgen, F., Alais, C., Fiat, A. M., \& Jollès, P. (1972a). Studies on primary structure of cow kappa-casein-primary sequence of cow para-kappa-casein. Chimia, 26(12), 645-646.

Jollès, J., Schoentgen, F., Alais, C., Fiat, A. M., \& Jollès, P. (1972b). Studies on the primary structure of cow kappa-casein: Structural features of para-kappa-casein; N-terminal sequence of kappa-caseinoglycopeptide studied with a sequencer. Helvetica Chimica Acta, 55(8), 2872-2883. PMid:4653404. http://dx.doi.org/10.1002/hlca.19720550820

Jones, R. G., Ober, C. K., Hodge, P., Kratochvíl, P., Moad, G., \& Vert, M. (2012). Terminology for aggregation and selfassembly in polymer science (IUPAC Recommendations 2013). Pure and Applied Chemistry, 85(2), 463-492. http://dx.doi.org/10.1351/PAC-REC-12-03-12

Kanamori, M., Kawaguchi, N., Ibuki, F., \& Doi, H. (1980). Attachment sites of carbohydrate moieties to peptide chain of bovine kappa-casein from normal milk. Agricultural and Biological Chemistry, 44(8), 1855-1861.

Krieger, I. M., \& Dougherty, T. J. (1959). A mechanism for non-Newtonian flow in suspensions of rigid spheres. Transactions of the Society of Rheology, 3(1), 137-152. http://dx.doi.org/10.1122/1.548848

Kruif, C. G. (1998). Supra-aggregates of casein micelles as a prelude to coagulation. Journal of Dairy Science, 81(11), 30193028. http://dx.doi.org/10.3168/jds.S0022-0302(98)75866-7

Kruif, C. G. (1999). Casein micelle interactions. International Dairy Journal, 9(3), 183-188. http://dx.doi.org/10.1016/S09586946(99)00058-8

Kruif, C. G. (2014). The structure of casein micelles: A review of small-angle scattering data. Journal of Applied Crystallography, 47(5), 1479-1489. http://dx.doi.org/10.1107/S1600576714014563

Kruif, C. G., \& Holt, C. (2003). Casein micelle structure, functions and interactions. In P. L. H. McSweeney \& P. F. Fox (Eds.), Advanced dairy chemistry (Vol. 1 Proteins, 3rd ed., pp. 233-276). Boston: Springer US. http://dx.doi.org/10.1007/978-1-44198602-3_5

Kruif, C. G., \& Huppertz, T. (2012). Casein micelles: Size distribution in milks from individual cows. Journal of Agricultural and Food Chemistry, 60(18), 4649-4655. PMid:22486748. http://dx.doi.org/10.1021/jf301397w

Kruif, C. G., Huppertz, T., Urban, V. S., \& Petukhov, A. V. (2012). Casein micelles and their internal structure. Advances in Colloid and Interface Science, 171-172, 36-52. PMid:22381008. http://dx.doi.org/10.1016/j.cis.2012.01.002

Leclerc, E., \& Calmettes, P. (1997). Structure of $\beta$-casein micelles. Physica B, Condensed Matter, 241-243, 1141-1143. http://dx.doi.org/10.1016/S0921-4526(97)00850-8

Lucey, J. A., \& Horne, D. S. (2009). Milk salts: Technological significance. In P. L. H. McSweeney \& P. F. Fox (Eds.), Advanced dairy chemistry (Vol. 3, 3rd ed., pp. 351-389). Boston: Springer US. http://dx.doi.org/10.1007/978-0-387-84865-5_9

Marchin, S., Putaux, J., Pignon, F., \& Leonil, J. (2007). Effects of the environmental factors on the casein micelle structure studied by cryo transmission electron microscopy and small-angle x-ray scattering/ultrasmall-angle x-ray scattering. The Journal of Chemical Physics, 126(4), 45101. PMid:17286511. http://dx.doi.org/10.1063/1.2409933

McGann, T. C. A., Kearney, R. D., Buchheim, W., Posner, A. S., Betts, F., \& Blumenthal, N. C. (1983). Amorphous calcium phosphate in casein micelles of bovine milk. Calcified Tissue International, 35(6), 821-823. PMid:6652558. http://dx.doi.org/10.1007/BF02405131

McMahon, D. J., \& Brown, R. (1984). J. Composition, structure, and integrity of casein micelles: A review. Journal of Dairy Science, 67(3), 499-512. http://dx.doi.org/10.3168/jds.S0022-0302(84)81332-6 
Mercier, J. C., Brignon, G., \& Ribadeau-Dumas, B. (1973). Structure primaire de la caseine kappaB bovine: Sequence complete. European Journal of Biochemistry, 35(2), 222-235. PMid:4577852. http://dx.doi.org/10.1111/j.14321033.1973.tb02829.x

Mercier, J. C., Grosclaude, F., \& Ribadeau-Dumas, B. (1971). Structure primaire de la caseine alphasl-bovine: Sequence complete. European Journal of Biochemistry, 23(1), 41-51. PMid:4331376. http://dx.doi.org/10.1111/j.14321033.1971.tb01590.x

Mercier, J. C., Uro, J., Ribadeau-Dumas, B., \& Grosclaude, F. (1972). Structure primaire du caséinomacropeptide de la caséine kB1 bovine. European Journal of Biochemistry, 27(3), 535-547. PMid:4559180. http://dx.doi.org/10.1111/j.14321033.1972.tb01870.x

Mezzenga, R., \& Fischer, P. (2013). The self-assembly, aggregation and phase transitions of food protein systems in one, two and three dimensions. Reports on Progress in Physics, 76(4), 46601. PMid:23455715. http://dx.doi.org/10.1088/00344885/76/4/046601

Morr, C. V. (1967). Effect of oxalate and urea upon ultracentrifugation properties of raw and heated skimmilk casein micelles. Journal of Dairy Science, 50(11), 1744-1751. http://dx.doi.org/10.3168/jds.S0022-0302(67)87710-5

Nöbel, S., Kern, C., Sonne, A., Bähler, B., \& Hinrichs, J. (2016). Apparent voluminosity of casein micelles in the temperature range $35-70{ }^{\circ} \mathrm{C}$. International Dairy Journal, 59, 80-84. http://dx.doi.org/10.1016/j.idairyj.2016.03.010

Nöbel, S., Weidendorfer, K., \& Hinrichs, J. (2012). Apparent voluminosity of casein micelles determined by rheometry. Journal of Colloid and Interface Science, 386(1), 174-180. PMid:22918047. http://dx.doi.org/10.1016/j.jcis.2012.07.075

O'Mahony, J. A., \& Fox, P. F. (2014). Milk: An overview. In M. Boland, H. Singh \& A. Thompson (Eds.), Milk proteins (2nd ed., pp. 19-73). London: Elsevier.

Ouanezar, M., Guyomarc'h, F., \& Bouchoux, A. (2012). AFM imaging of milk casein micelles: Evidence for structural rearrangement upon acidification. Langmuir, 28(11), 4915-4919. PMid:22384811. http://dx.doi.org/10.1021/la3001448

Parker, T. G., \& Dalgleish, D. G. (1981). Binding of calcium ions to bovine $\beta$-casein. The Journal of Dairy Research, 48(1), 71 76. PMid:7264011. http://dx.doi.org/10.1017/S0022029900021476

Philippe, M., Le Graët, Y., \& Gaucheron, F. (2005). The effects of different cations on the physicochemical characteristics of casein micelles. Food Chemistry, 90(4), 673-683. http://dx.doi.org/10.1016/j.foodchem.2004.06.001

Pyne, G. T., \& McGann, T. C. A. (1960). The colloidal phosphate of milk: II. Influence of citrate. The Journal of Dairy Research, 27(1), 9-17. http://dx.doi.org/10.1017/S0022029900010074

Rambaran, R. N., \& Serpell, L. C. (2008). Amyloid fibrils: Abnormal protein assembly. Prion, 2(3), 112-117. PMid:19158505 http://dx.doi.org/10.4161/pri.2.3.7488

Ranadheera, C. S., Liyanaarachchi, W. S., Chandrapala, J., Dissanayake, M., \& Vasiljevic, T. (2016). Utilizing unique properties of caseins and the casein micelle for delivery of sensitive food ingredients and bioactives. Trends in Food Science \& Technology, 57, 178-187. http://dx.doi.org/10.1016/j.tifs.2016.10.005

Rasmussen, L. K., Højrup, P., \& Petersen, T. E. (1994). Disulphide arrangement in bovine caseins: Localization of intrachain disulphide bridges in monomers of $\mathrm{k}$ - and as2-casein from bovine milk. The Journal of Dairy Research, 61(4), 485-493. PMid:7829753. http://dx.doi.org/10.1017/S0022029900028417

Rose, D., \& Colvin, J. R. (1966). Appearance and size of micelles from bovine milk. Journal of Dairy Science, 49(9), 1091-1097. PMid:5950524. http://dx.doi.org/10.3168/jds.S0022-0302(66)88023-2

Saito, T., \& Itoh, T. (1992). Variations and distributions of O-glycosidically linked sugar chains in bovine k-casein. Journal of Dairy Science, 75(7), 1768-1774. PMid:1500573. http://dx.doi.org/10.3168/jds.S0022-0302(92)77936-3

Shimmin, P. D., \& Hill, R. D. (1964). An electron microscope study of the internal structure of casein micelles. The Journal of Dairy Research, 31(1), 121-123. http://dx.doi.org/10.1017/S0022029900017982

Slattery, C. W. (1976). Casein micelle structure; an examination of models. Journal of Dairy Science, 59(9), 1547-1556. PMid:987079. http://dx.doi.org/10.3168/jds.S0022-0302(76)84403-7

Slattery, C. W., \& Evard, R. (1973). A model for the formation and structure of casein micelles from subunits of variable composition. Biochimica et Biophysica Acta (BBA) - Protein Structure, 317(2), 529-538. PMid:19999736.

http://dx.doi.org/10.1016/0005-2795(73)90246-8

Stothart, P. H. (1989). Subunit structure of casein micelles from small-angle neutron-scattering. Journal of Molecular Biology, 208(4), 635-638. PMid:2810358. http://dx.doi.org/10.1016/0022-2836(89)90154-X

Stothart, P. H., \& Cebula, D. J. (1982). Small-angle neutron scattering study of bovine casein micelles and sub-micelles. Journal of Molecular Biology, 160(2), 391-395. PMid:7175936. http://dx.doi.org/10.1016/0022-2836(82)90185-1

Swaisgood, H. E. (2003). Chemistry of the caseins. In P. L. H. McSweeney \& P. F. Fox (Eds.), Advanced dairy chemistry (Vol. $1 \mathrm{~A}$, 3rd ed., pp. 139-201). Boston: Springer US. http://dx.doi.org/10.1007/978-1-4419-8602-3_3

Tran, V. D., \& Baker, B. E. (1970). Casein. IX. Carbohydrate moiety of K-casein. Journal of Dairy Science, 53(8), 1009-1012. PMid:5469729. http://dx.doi.org/10.3168/jds.S0022-0302(70)86336-6

Trejo, R., Dokland, T., Jurat-Fuentes, J., \& Harte, F. (2011). Cryo-transmission electron tomography of native casein micelles from bovine milk. Journal of Dairy Science, 94(12), 5770-5775. PMid:22118067. http://dx.doi.org/10.3168/jds.2011-4368

Treweek, T. M., Thorn, D. C., Price, W. E., \& Carver, J. A. (2011). The chaperone action of bovine milk aS1- and aS2-caseins and their associated form aS-casein. Archives of Biochemistry and Biophysics, 510(1), 42-52. PMid:21457703. http://dx.doi.org/10.1016/j.abb.2011.03.012 
Tuinier, R., \& Kruif, C. G. (2002). Stability of casein micelles in milk. The Journal of Chemical Physics, 117(3), 1290-1295. http://dx.doi.org/10.1063/1.1484379

Vreeman, H. J., Visser, S., Slangen, C. J., \& Van Riel, J. A. (1986). Characterization of bovine K-casein fractions and the kinetics of chymosin-induced macropeptide release from carbohydrate-free and carbohydrate-containing fractions determined by high-performance gel-permeation chromatography. The Biochemical Journal, 240(1), 87-97. PMid:3103611. http://dx.doi.org/10.1042/bj2400087

Walstra, P. (1999). Casein sub-micelles: Do they exist? International Dairy Journal, 9(3), 189-192. http://dx.doi.org/10.1016/S0958-6946(99)00059-X

Walstra, P. (2002). Physical chemistry of foods. Boca Raton: CRC Press. http://dx.doi.org/10.1201/9780203910436

Waugh, D. F., \& Von Hippel, P. H. (1956). k-Casein and the stabilization of casein micelles. Journal of the American Chemical Society, 78(18), 4576-4582. http://dx.doi.org/10.1021/ja01599a017

White, J. C. D., \& Davies, D. T. (1958). 712. The relation between the chemical composition of milk and the stability of the caseinate complex: I. General introduction, description of samples, methods and chemical composition of samples. The Journal of Dairy Research, 25(2), 236-255. http://dx.doi.org/10.1017/S0022029900009249

Financiamento: Nenhum. 